\title{
COMPLEMENTARY SURFACES FOR A VECTOR FIELD
}

\section{T. K. PAN}

1. Introduction. Let $v$ be a vector field in a surface in an ordinary space. The author defined the curve of $v$ and the asymptotic line of $v$ and proved that, at a point on a nondevelopable surface, they are curves along each of which the straight lines on $v$ form generators of a developable surface [2]. Corresponding to each family of these curves, there is one family of developable surfaces. They are called developable surfaces of $v$. The family of developable surfaces of $v$, which are formed by straight lines on $v$ along the curves of $v$ on a surface, evidently has the surface itself as locus of edges of regression. In this paper, the locus of edges of regression of the other family of developable surfaces of $v$ is investigated. Unless otherwise indicated, by a family of developable surfaces of $v$ is always meant this second family of developable surfaces, that is, the family of developable surfaces formed by straight lines on $v$ along the asymptotic lines of $v$. Orthocenter of associate curvature of $v$ is defined to include as a special case the center of geodesic curvature of an orthogonal trajectory of a geodesic family. Complementary surfaces for a geodesic family according to Bianchi are generalized into complementary surfaces for a vector field. Properties of these complementary surfaces and related curves are studied.

The notation of Eisenhart [1] will be used for the most part except that $\Gamma_{\beta \gamma}^{\alpha}$ is used for the Christoffel symbol of the second kind.

2. Orthocenter of associate curvature. Let $S: x^{i}=x^{i}\left(u^{1}, u^{2}\right)$, $i=1,2,3$, be a real proper analytic surface in an ordinary space with reference to a rectangular cartesian coordinate system. Unless explicitly stated, by a surface is always meant a nondevelopable surface. Associate to each point of $S$ an arbitrary but fixed unit vector $v^{i}$ such that $v^{i}=p^{\alpha} x^{i}, \alpha, g_{\alpha \beta} p^{\alpha} p^{\beta}=1, \alpha, \beta=1,2$, and $v^{i}$ are real and single-valued functions of $u^{\alpha}$ of class 1 . It will be denoted by $v$ or $p$ at appropriate places.

Let $C: u^{\alpha}=u^{\alpha}(s)$ be an analytic curve on $S$, where $s$ is its arc length. The normal component of the derived vector of $v$ along $C$ is called the normal curvature vector of $v$ along $C$, whose magnitude is the normal curvature of $v$ along $C$. An asymptotic line of $v$ on $S$ is defined as a curve along which the normal curvature of $v$ is zero, and a curve of $v$

Presented to the Society, February 27, 1954; received by the editors June 1, 1954. 
on $S$ is defined as a curve to which the vectors $v$ are tangent. They can be represented respectively by the following equations:

$$
d_{\alpha \beta} p^{\alpha} d u^{\beta}=0, \quad \epsilon_{\alpha \beta} p^{\alpha} d u^{\beta}=0 .
$$

It is found that the asymptotic lines of $v$ form a conjugate net with the curves of $v$ and form an orthogonal net with the lines of curvature of $v$, which are curves on $S$ with principal direction of $v$ at each and every point - a direction with respect to which the normal curvature of $v$ has extreme value [2, pp. 956-958].

The orthogonal trajectories of the curves of $v$ form a family of curves on $S$ defined by

$$
g_{\alpha \beta} p^{\alpha} d u^{\beta}=0 .
$$

For simplicity, they will be called the orthogonal trajectories of $v$. The reciprocal of the associate curvature of $v$ at a point $P$ along a curve $C$ is called the radius of associate curvature of $v$ at $P$ along $C$ and is denoted by ${ }_{v} r_{g}$. The point which lies on the straight line through $P$ in the direction of $v$ at $P$ and which is at a distance $-{ }_{v} r_{\theta} \cos \theta$ from $P$, where $\theta$ is the angle at $P$ between $C$ and the orthogonal trajectory of $v$, is called the orthocenter of associate curvature of $v$ at $P$ along $C$. The negative sign and the term "ortho" are used in the above definition because of the fact that the associate curvature of a unit normal to $v$ at $P$ along $C$ is the negative of that of $v$ at $P$ along $C[1, \mathrm{p} .199]$. We shall need

TheOREM 2.1. The center of geodesic curvature at a point of an orthogonal trajectory of a family of geodesics on a surface is coincident with the orthocenter at the point of the field of unit tangent vectors to the geadesics along the asymptotic line of the field.

The proof is as follows. Let $u^{2}=$ const. be a family of geodesics on $S$. Then it is necessary and sufficient that $\Gamma^{2}{ }_{11}=0[1, \mathrm{p} .172]$. Take these geodesics as curves of a unit vector field $v$. Consequently, we have

$$
p^{1}=1 /\left(g_{11}\right)^{1 / 2}, \quad p^{2}=0 .
$$

Let $u^{1}=$ const. be the asymptotic lines of $v$. Then $d_{12}=0$. From (2.1) and (2.2), the asymptotic directions of $v$ and the directions of the orthogonal trajectories of $v$ are found to be

respectively.

$$
\begin{aligned}
d u^{1} / d s & =0, \quad d u^{2} / d s=-1 /\left(g_{22}\right)^{1 / 2} \text { and } \\
\frac{d u^{1}}{d s} & =\frac{g_{12}}{\left(g_{11} g\right)^{1 / 2}}, \quad \frac{d u^{2}}{d s}=\frac{-g_{11}}{\left(g_{11} g\right)^{1 / 2}},
\end{aligned}
$$


Let ${ }_{v} \kappa_{v}$ be the associate curvature of $v$ along the asymptotic line of $v$ at a point $P$. Then we have

$$
\frac{\Gamma_{12}^{2}}{\left(g_{11} g_{22}\right)^{1 / 2}}=\frac{{ }_{v} \kappa_{g} g_{11}}{\left(g_{11} g\right)^{1 / 2}}
$$

which gives

$$
{ }_{{ }^{\prime}} r_{g}=\frac{g_{11}\left(g_{22}\right)^{1 / 2}}{g^{1 / 2} \Gamma^{2}{ }_{12}} .
$$

Let $\theta$ be the angle at $P$ between the orthogonal trajectory of $v$ and the asymptotic line of $v$. Then from (2.4) we have

$$
\cos \theta=g^{1 / 2} /\left(g_{11} g_{22}\right)^{1 / 2}
$$

which together with (2.5) gives

$$
-{ }_{{ }} r_{\theta} \cos \theta=-\left(g_{11}\right)^{1 / 2} / \Gamma^{2}{ }_{12} \text {. }
$$

Let $\kappa_{g}$ be the geodesic curvature of the orthogonal trajectory of $v$ at $P$. By (2.3) and (2.4) we find

$$
\Gamma_{12}^{2} \cdot \frac{1}{\left(g_{11}\right)^{1 / 2}} \cdot \frac{-g_{11}}{\left(g_{11} g\right)^{1 / 2}}=-\kappa_{g} \cdot \frac{-g_{11}}{\left(g_{11} g\right)^{1 / 2}}
$$

which gives from (2.6)

$$
r_{o}=\frac{1}{\kappa_{g}}=-{ }_{{ }_{v}} r_{g} \cos \theta
$$

Thus Theorem 2.1 is established.

It is evident that the curves of a vector field form a family of geodesics on $S$ if and only if the curves of the field coincide with the indicatrices of the field, which are curves on $S$ relative to which the vectors of the field are parallel in the sense of Levi-Civita. It is also clear from Theorem 2.1 that the orthocenter at a point of a vector field along the asymptotic line of the field is a suitable generalization of the center of geodesic curvature at the point of the orthogonal trajectory of a family of geodesics on a surface [1, p. 275].

3. Complementary surface. A field of vectors on a surface $S$, whose directions at each and every point of $S$ constitute a family of asymptotic directions of $S$, is called an asymptotic vector field, or an asymptotic field, of $S$. A vector field $v$ is an asymptotic field if and only if the family of asymptotic lines of $v$ and the family of curves of $v$ are identical. Hence, the two families of developable surfaces of an asymptotic 
field on $S$ are not distinct and the surface $S$ itself is the locus of their edges of regression. Unless otherwise stated, it is assumed in the following discussion that $v$ is not an asymptotic field.

Let $\bar{P}\left(\bar{x}^{i}\right)$ defined by

$$
\bar{x}^{i}=x^{i}+\rho p^{\alpha} \partial x^{i} / \partial u^{\alpha}
$$

be any point of the edge of regression of a developable surface of the family of developable surfaces of $v$, which corresponds to $P\left(x^{i}\right)$ of the related asymptotic line of $v$. Then $d \bar{x}^{i} / d s$ are proportional to $p^{\alpha} \partial x^{i} / \partial u^{\alpha}$ and we have

$$
\frac{d x^{i}}{d s}+\rho \frac{d}{d s}\left(p^{\alpha} \frac{\partial x^{i}}{\partial u^{\alpha}}\right)+p^{\alpha} \frac{\partial x^{i}}{\partial u^{\alpha}} \frac{d \rho}{d s}=h p^{\alpha} \frac{\partial x^{i}}{\partial u^{\alpha}} .
$$

Multiplying (3.1) by $p^{\beta} \partial x^{i} / \partial u^{\beta}$ and summing up $i$, we obtain

$$
g_{\alpha \beta} p^{\beta} \frac{d u^{\alpha}}{d s}+\frac{d \rho}{d s}=h
$$

since

$$
\begin{gathered}
\frac{d x^{i}}{d s}=\frac{\partial x^{i}}{\partial u^{\alpha}} \frac{d u^{\alpha}}{d s}, \quad \sum_{i} p^{\alpha} \frac{\partial x^{i}}{\partial u^{\alpha}} \frac{d}{d s}\left(p^{\beta} \frac{\partial x^{i}}{\partial u^{\beta}}\right)=0, \\
\sum_{i}\left(p^{\alpha} \frac{\partial x^{i}}{\partial u^{\alpha}}\right)\left(p^{\beta} \frac{\partial x^{i}}{\partial u^{\beta}}\right)=g_{\alpha \beta} p^{\alpha} p^{\beta}=1 .
\end{gathered}
$$

Substituting (3.2) into (3.1) and simplifying, we obtain

$$
\frac{d x^{i}}{d s}+\rho \frac{d}{d s}\left(p^{\alpha} \frac{\partial x^{i}}{\partial u^{\alpha}}\right)=g_{\alpha \beta} p^{\alpha} \frac{d u^{\beta}}{d s} p^{\gamma} \frac{\partial x^{i}}{\partial u^{\gamma}} .
$$

Multiplying (3.3) by $d x^{i} / d s$, summing over $i$, noting that ${ }_{v} K={ }_{v} \kappa_{g}$ since ${ }_{v} \kappa_{n}=0$, and simplifying, we have

$$
\rho=-{ }_{v} r_{g} \cos \theta
$$$$
(\theta \neq \pi / 2) \text {. }
$$

Hence the coordinates of $\bar{P}$ on the edge of regression are given by

$$
\bar{x}^{i}=x^{i}-{ }_{v} r_{g} \cdot \cos \theta \cdot p^{\alpha} \frac{\partial x^{i}}{\partial u^{\alpha}}
$$

which represent exactly the orthocenter of $v$ along the asymptotic line of $v$ at $P$.

Equations (3.5) represent the edge of regression when $P\left(x^{i}\right)$ moves on an asymptotic line of $v$, that is, when $x^{i}=x^{i}(s)$, where $s$ is its arc length; (3.5) represent a surface $\bar{S}$ when the above asymptotic 
line of $v$ assumes all positions of the family of asymptotic lines of $v$, that is, when $x^{i}=x^{i}\left(u^{1}, u^{2}\right)$. Thus $\bar{S}$ is formed by the edges of regression of the family of developable surfaces of $v$. Following Bianchi we call $\bar{S}$ the surface complementary to $S$ for the vector field $v$. Hence we have

TheOREM 3.1. A complementary surface of a surface $S$ for a vector field is the locus of the orthocenters of the vector field along the asymptotic lines of the vector field on $S$ which determines the complementary surface.

When the family of curves of a vector field is a family of geodesics on $S$, Theorem 2.1 and Theorem 3.1 show immediately that the complementary surface of $S$ for a vector field reduces to Bianchi's complementary surface of $S$ for a geodesic family. From this point of view complementary surfaces for a vector field are generalizations of Bianchi's complementary surfaces for a geodesic family.

We know from (3.4) that $\rho$ is undefined when ${ }_{v} \kappa_{g}=0$. Since $S$ is assumed to be nondevelopable, this happens if and only if the family of asymptotic lines of $v$ is identical with the family of indicatrices of $v$ $\left[2\right.$, p. 962]..$^{1}$ We also note from (3.4) that $\rho=0$ if and only if ${ }_{v} r_{g}=0$. This case implies ${ }_{v} K_{v}={ }_{v} \kappa=\infty$, which is excluded by the assumption that $v^{i}$ are functions of class 1 . Hence, we have

THEOREM 3.2. The complementary surface of a surface for a vector field is undefined if and only if the family of asymptotic lines of the field is identical with the family of indicatrices of the field. A surface is selfcomplementary for a vector field if and only if the field is an asymptotic field of the surface.

Since the straight lines on $v$ along a curve of $v$ form a developable surface with the curve of $v$ as edge of regression, it is obvious that any surface is self-complementary for any vector field along a curve of the field.

The complementary surface $\bar{S}$ of $S$ for a vector field $v$ is degenerate if and only if $h$ in (3.1) is zero. From (3.2) we have

that is,

$$
\sin \theta+d \rho / d s=0,
$$

$$
\rho=-\int \sin \theta d s
$$

1 The author takes this opportunity to correct some errors in the paper [2]: p. 962 , line 18 , for ${ }_{v} k=0$ " read " ${ }_{v} k \cdot{ }_{v} \kappa_{n}=0 "$; p. 962, line 9 , add ${ }^{*}{ }_{v} k_{n}=0$ if and only if $C$ is an asymptotic line of the field;" p. 966 , line 3 , for "in $V_{n}$." read "in $V_{n}$ is the absolute curvature in $V_{m}$ of the field with respect to the indicatrix of the field in $V_{n}$." 
Hence, by Theorem 2.1 and equation (3.6), we obtain

THEOREM 3.3. A necessary and sufficient condition that the complementary surface of a surface for a vector field be degenerate is that the orthogonal trajectories of the field are curves with radius of geodesic curvature equal to $-\int \sin \theta d s$.

Let $v$ be a principal field of $S$, which is a field of vectors whose directions at each and every point of $S$ constitute a family of principal directions of $S$. Then the other family of lines of curvature of $S$ becomes the family of asymptotic lines of $v$ on $S$ [2, p. 958]. Consequently, $\sin \theta=0$. Hence, if the complementary surface of $S$ for a principal field is degenerate, the family of lines of curvature of $S$ distinct from the field consists of curves of constant geodesic curvature. Conversely, if a family of lines of curvature of $S$ consists of curves of constant geodesic curvature different from zero, the complementary surface of $S$ for the other family of lines of curvature of $S$ is degenerate. Hence we have

TheOREM 3.4. A necessary and sufficient condition that the tangents to a family of lines of curvature of $S$ at points of a curve of the other family of lines of curvature of $S$ form a cone is that the latter family consists of curves of constant geodesic curvature different from zero.

For example, the meridians and parallels of a surface of revolution form two families of lines of curvature of the surface. It is evident geometrically that the tangents to the meridians at points of a parallel form a cone. Hence, by Theorem 3.4, the parallels on a surface of revolution are curves of constant geodesic curvature. This result can be verified analytically and is an established fact $[1$, p. 192, Ex. 1].

On a developable surface, the lines of curvature are its generators and their orthogonal trajectories. The tangents to one family of lines of curvature at points of a curve of the other family of lines of curvature form either a plane or the developable surface itself.

4. Reciprocal relation. Consider a curve on a surface and the tangent planes to the surface at points of the curve. The envelope of this one-parameter family of tangent planes is a developable surface. Through each point of the curve there passes a generator of the developable surface. The direction of the generator and that of the tangent to the curve at the point are conjugate. If the family of asymptotic lines of a vector field is taken as the family of curves of another vector field, then the family of curves of the first vector field 
is the family of asymptotic lines of the second vector field. Hence, by Theorem 3.1, we have

THEOREM 4.1. The tangents to one family of curves of a conjugate net of curves on a surface at points of a curve of the other family form a developable surface. The edge of regression of the developable surface is the locus of orthocenters of associate curvature of the former family at points of that curve of the latter family.

Let $\bar{S}$, the complementary surface of $S$ for a vector field $v$, be proper and distinct from $S$. Let $\mathfrak{\&}$ be the family of curves of $v$ on $S$ and $\mathfrak{A}$, the family of asymptotic lines of $v$ on $S$. Let $\bar{\imath}$ denote the edges of regression of the family of developable surfaces $D$ of $v$, which form a family of curves on $\bar{S}$. Let $\overline{\mathfrak{A}}$ be a family of curves which together with $\overline{\mathfrak{R}}$ forms a conjugate net on $\bar{S}$. It is clear from definition that one developable surface in $D$, say $D_{1}$, is associated with one asymptotic line of $v$ in $\mathfrak{A}$, say $\mathfrak{A}_{1}$, and with one edge of regression in $\overline{\mathfrak{R}}$, say $\overline{\mathfrak{R}}_{1}$. $D_{1}$ is the envelope of the tangent planes to $S$ at points of $\mathfrak{A}_{1}$, which in turn are osculating planes of $\overline{\mathfrak{l}}_{1}$ on $\bar{S}$. The generators of $D_{1}$ are common tangents of $S$ and $\bar{S}$, whose points of contact $P$ and $\bar{P}$, which lie on $\mathfrak{A}_{1}$ and $\overline{\mathfrak{R}}_{1}$ respectively, are corresponding points on $S$ and $\bar{S}$. Since the tangents to a curve in $\mathfrak{R}$ are tangent to $\bar{S}$, the developable surface generated by them is an envelope of a family of tangent planes to $\bar{S}$ at points $\bar{P}$ of a curve in $\overline{\mathfrak{A}}$. This developable surface evidently belongs to that family of developable surfaces of $v$ which are formed by straight lines on $v$ along the curves of $v$ on $S$. Since $\bar{\Omega}$ on $\bar{S}$ is determined by the vector field $v$ on $S$, we may call $\bar{\Omega}$ the family of curves of $v$ on $\bar{S}$. Consequently $S$ is the complementary surface of $\bar{S}$ for $v$. Hence, we have

TheOREM 4.2. Complementary relation between two surfaces $S$ and $\bar{S}$ for a vector field is reciprocal. The osculating planes of the family of curves of the vector field on one surface are tangent to the other surface along the family of asymptotic lines of the vector field. The orthocenters of associate curvature of the family of curves of $v$ on $S$ and of the family of curves of $v$ on $\bar{S}$ are corresponding points on $\bar{S}$ and $S$ respectively, corresponding points being the points of tangency of a common tangent to the two surfaces.

The second part of the above theorem is a generalization of a theorem of Beltrami [1, p. 275].

Viewed from the enveloping space, the curves in $\mathfrak{l}$ on $S$ can be represented by $d x^{i} / d s=p^{\alpha} \partial x^{i} / \partial u^{\alpha}$, from which we obtain 


$$
\frac{d^{2} x^{i}}{d s^{2}}=p^{\alpha},{ }_{\gamma} p^{\gamma} \frac{\partial x^{i}}{\partial u^{\alpha}}+d_{\beta \gamma} p^{\beta} p^{\gamma} X^{i}
$$

where $X^{i}$ are the components of unit normal to $S$. Define a unit vector $\lambda^{i}$ such that

$$
\kappa \lambda^{i}=p^{\alpha}, \gamma p^{\gamma} \frac{\partial x^{i}}{\partial u^{\alpha}}+d_{\beta \gamma} p^{\beta} p^{\gamma} X^{i}
$$

where $\kappa$ is the absolute curvature of the curves on $\&$ of $S$ in the enveloping space. Then the curves in $\&$ on $S$ are union curves corresponding to a congruence of unit vectors which can be represented either by $\lambda^{i}$ defined in (4.1) or by any linear combination $m \lambda^{i}+n v^{i}$ with $m \neq 0$. Since there are advantages to using vectors orthogonal to $v^{i}$, we prefer and assume the congruence of such unit vectors as $\lambda^{i}$ for expressing the curves in $\mathfrak{l}$ as union curves on $S$. When $\mathfrak{l}$ consists of a family of geodesics on $S$, we have $p^{\alpha}{ }_{, \gamma} p^{\gamma}=0$ and the $\lambda^{i}$ become identical with $X^{i}$ as is expected. Thus the curves of $v$ on $S$ constitute a family of union curves on $S$ relative to the congruence $\lambda^{i}$ in the enveloping space.

Let $F$ be a surface. Let a straight line in the enveloping space be associated with each point of $F$ such that the direction cosines of the straight line, which is not tangent to $F$, and their first partial derivatives are continuous. There is a congruence of straight lines' like this associated with $F$. C. E. Springer found that the developables of the congruence intersect $F$ in a net of curves called the intersector net $[3$, p. 992]. Consequently we have

TheOREM 4.3. The edges of regression of the developables of a congruence in space associated with a surface $F$ along one family of the intersector net are union curves on the surface $S$ which is the locus of these edges. The developables of the congruence along the other family of the intersector net envelope this surface $S$ along the asymptotic lines of the vector field whose curves are identical with the union curves.

\section{REFERENCES}

1. L. P. Eisenhart, An introduction to differential geometry with the use of the tensor calculus, Princeton University Press, 1947.

2. T. K. Pan, Normal curvature of a vector field, Amer. J. Math. vol. 74 (1952) pp. 955-966.

3. C. E. Springer, Rectilinear congruences whose developables intersect a surface in its lines of curvature, Bull. Amer. Math. Soc. vol. 51 (1945) pp. 990-996.

UNIVERSITY OF OKLAHOMa 